\title{
ECVlab: A Web-Based Virtual Laboratory System for Electronic Circuit Simulation
}

\author{
Ouyang Yang, Dong Yabo, Zhu Miaoliang, \\ Huang Yuewei, Mao Song, and Mao Yunjie \\ Lab of Networking Center, Collage of Computer Science and Technology \\ Zhejiang University, Hangzhou 310027, P.R.China \\ \{lily, dongyb, zhum, yuewei, mason, myjesky\}@zju.edu.cn
}

\begin{abstract}
In this paper, we describe the design and implementation of a web-based electronic circuit simulation system named ECVlab. This system combines technologies as rich client technology, XML, and circuit simulation, and provides the user with vivid interface, convenient operation and powerful simulation capability. At the moment, ECVlab has been implemented and successfully applied in an undergraduate course in Zhejiang University and it was evaluated by undergraduate students. Students made statistically significant learning gains as a result of using ECVlab, and rated them positively in terms of improving operation capability, enhancing interests for digital circuit and offering opportunity to inspire innovation and exploration.
\end{abstract}

\section{Introduction}

Nowadays, there is an increasing interest in web-based education for its prominent advantages of system opening and resources reusability. At universities, electronic circuit experiment is a vital part of higher education since it is a valuable method of learning which gives a learner the feeling of involvement. It can practice students' operation ability and also provide a good opportunity for them to put into practice what they've learned in class. And the learning process can be best facilitated if tools and models are freely and widely available, not just in the dedicated laboratories. Web-based simulation environments, combining distance education, group training and real-time interaction, can serve as a good approach.

Simulation is a process, during which experiments are conducted on a designed real system model for the purpose of understanding the behavior of the system or evaluating various strategies for the operation of the system. The power of simulation is the ability to model the dynamics of a real system and to analyze the results $[6$. Meanwhile, the availability and interactive nature of web-based simulation serves as a good medium for students to experience the complexity of collaborative work.

This paper is to present a web-based virtual laboratory system for electronic circuit simulation (ECVlab). In this system, students can conduct experiment 
through web browser using virtual apparatuses like multimeter and equipments like resistance and tolerance.

The rest of this paper is organized as follows. In section 2, we list some related Web-based simulations. The design and structure of the vlab system is specified in section 3. In section 4, we demonstrate an example of applying our system. Later, an analysis of survey in undergraduate students is presented in section 5. Finally, in section 6 , we draw conclusions of this work and point out some directions of future work.

\section{Related Works}

Web-based simulation represents the combination of rapidly-developing internet $\&$ multimedia technology and simulation science. The ability of web technologies enhances the power of simulation in that web can service large simulation communities and allow developers to distribute models and simulations to end users. Since Fishwick 4] started to explore Web-based simulation as a new research area in the field of simulation, many Web-based simulation models and support systems have been developed.

Kuljis and Paul [2] introduced a variety of new technologies for discrete-event Web-based simulation technologies and reviewed related environments and languages too. John C. Waller and Natalie Foster [1] designed a virtual GC-MS (Gas Chromatography / Mass Spectrometry) by means of copying presentation of monitor to the screen. Students can operate this virtual instrument via web and the real instrument can be used to take more valuable experiment. Cheng K.W.E. et.al 3] demonstrated how a power electronics experiment is programmed in a remotely controlled laboratory setup. Huang and Miller [5] presented a prototype implementation of a Web-based federated simulation system using Jini and XML.

Some of the above simulation systems operate the experimental equipment through remote control and monitor by web-based tools. Due to the involvement of real equipment, limitation of cooperation and risk of damaging certain equipment still exist. The application of Java development tools and JavaBeans technology are widely used in several simulation systems. However, the interface developed by java applet is lack of intuitive and vivid expression. The major contribution of our work is to design a rich client simulation system which is totally independent of real experiment environment.

\section{ECVlab System}

\subsection{Design Objectives}

The ECVlab system is based on the project supported by a grant from the National Key Technologies R \& D Program of China. As an assistant experiment education system which plays an important role in distance-learning, it should be provided with characteristics such as relatively low requirement of system 
resources, powerful collaboration capability between user and server, platform flexibility, high performance of transform and communication on internet and so on. Furthermore, the system should provide students with real expression.

\subsection{System Architecture}

The ECVlab system uses an Browser/Server system architecture, which is shown in figure 1.

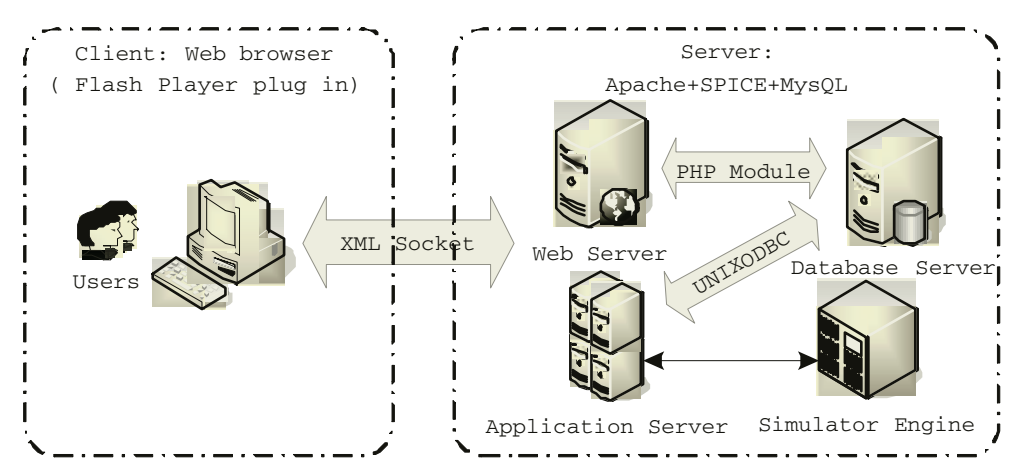

Fig. 1. ECVlab System Architecture

The server side runs on Linux system and can be divided into 3 parts: web server, application server and database server. The web server is responsible for user authentication, establishment of the virtual experiment framework, maintenance of user information; the application server manages the communication with multiple online flash client users, transfers parsed or encapsulated data to the spice simulator and return the results to client side. In ECVlab, information about user and experiments are stalled in database server, while the simulation logic is contained in application server.

On the server side, simulator engine plays an important roll in the whole system and we utilize SPICE (Simulation Program with Integrated Circuit Emphasis) to meet our simulation requirements. SPICE is a general-purpose circuit simulation program for nonlinear dc, nonlinear transient, and linear ac analysis which enables server to simulate analog circuit and print output in ASCII text.

The client side consists of the web browser and the embedded flash. Flash is a multimedia graphics program especially for use on the web. Compared with Animated images and Java applets, which are often used to create dynamic effects on Web pages, the advantages of Flash are:

- Flash loads much faster than animated images

- Flash allows interactivity, whereas animated images do not

- Flash can create a real scene, whereas Java applets can't

- Flash edition 2004 supports the object-oriented technology which enables us to develop powerful interactive web animation 
With these features, we use Flash to construct the virtual experiment environment. More detailed discussion about the interface will be shown in section 4. When a user starts an experiment, the client side firstly logins to the server through web browser and after certification, the user logins to the ECVlab system. After entering certain experiment, virtual experiment environment downloads automatically from the web browser and runs. During the experiment process, the environment consistently communicates with the application server to exchange data and display the simulation results.

\subsection{Communication Protocol}

An expandable and flexible communication protocol between server and client plays an important role in a well-designed web-based Vlab system. In our system, the protocol is used to transport following information:

- 1) Information about experiment and user, such as user name and password, experiment id and so on,

- 2) Parameters of components like value of resistance and apparatuses like pace of oscillograph,

- 3) Simulation results returned from simulator engine.

We use XML to construct the communication protocol. XML is a markup language for documents containing structured information and is designed to describe data that allows the author to define his own tags document structure. With this powerful data-exchange language, we can construct our own protocol by defining tags related to parameters of apparatus and simulation results. The form of ECVlab communication protocol is shown in figure 2.

The communication model of the ECVlab is shown in figure 3. The ECVlab system can be conveniently layered into four abstract levels. When the circuit

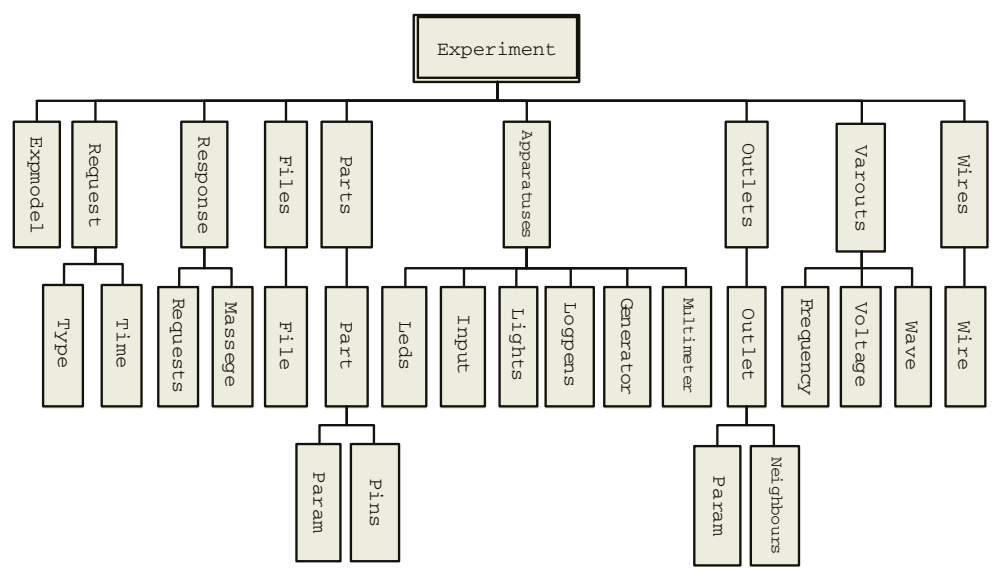

Fig. 2. ECVlab Communication Protocol 


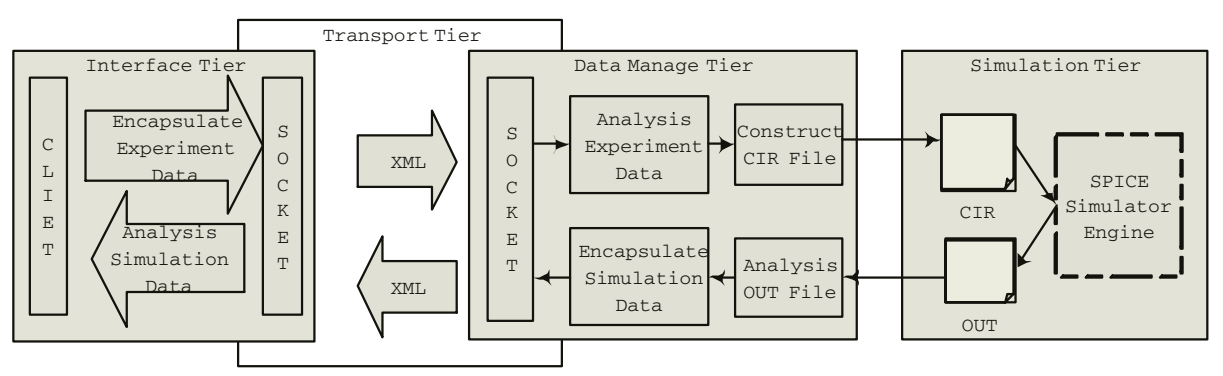

Fig. 3. Communication Mechanism of ECVlab

began to simulate, the circuit description file which contained circuit parameters and control information was encapsulated in XML file and transferred from interface layer to the data manage layer by socket. Later the submitted data was transferred into the formatted data needed by simulator engine and created a SPICE supported input file (.cir). After the simulator engine worked out the results and stored them in a .out file, the data management layer was responsible for analyzing the output and encapsulating required information into XML file similarly. Finally, the client converted the results into the form shown in apparatus such as the waveform shown in oscillograph and the voltage value in multimeter.

\section{An Example of Using the ECVlab}

We select one experiment of analog circuit in the undergraduate courses named Operation Amplifier (OPAMP). Through this experiment, students learn to use operation amplifier, resistance and capacitor to construct analog operation circuit and analyze the function of this circuit. In figure 4, the schematic diagram of OPAMP is shown in a), and b) demonstrates the equivalent circuit built by user in the virtual circuit environment.

Figure 5 shows a snapshot of the interface in ECVlab and demonstrates simulation results of the OPAMP. On the left hand, the system displays the tool bar which contains buttons for different operations. When a user starts to construct a circuit, he can add necessary components with appointed value to the circuit from the tool bar. For example, in OPAMP, user can add one operation amplifier and several resistances. After that, user needs to connect lines between these components. ECVlab enables the user to add or delete lines from this virtual circuit board freely and change the color of lines to differentiate between each others. In order to modify the circuit parameters and obtain the results of the experiment, user should use several apparatuses such as multimeter, oscillograph, and signal generator. In our system, the user can choose which of these apparatuses to be displayed on screen, and can zoom out or zoom in or move around at will. Furthermore, all these virtual apparatuses are highly similar to the real ones and the user can operate the buttons on them to modify parame- 


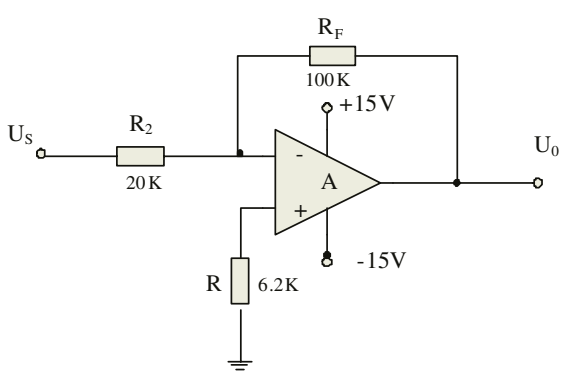

(a) Schematic Circuit Diagram

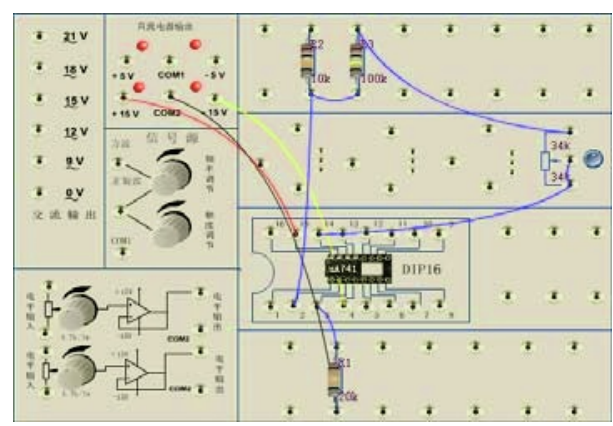

(b) Equivalent Virtual Circuit

Fig. 4. OPAMP Electronic Circuit

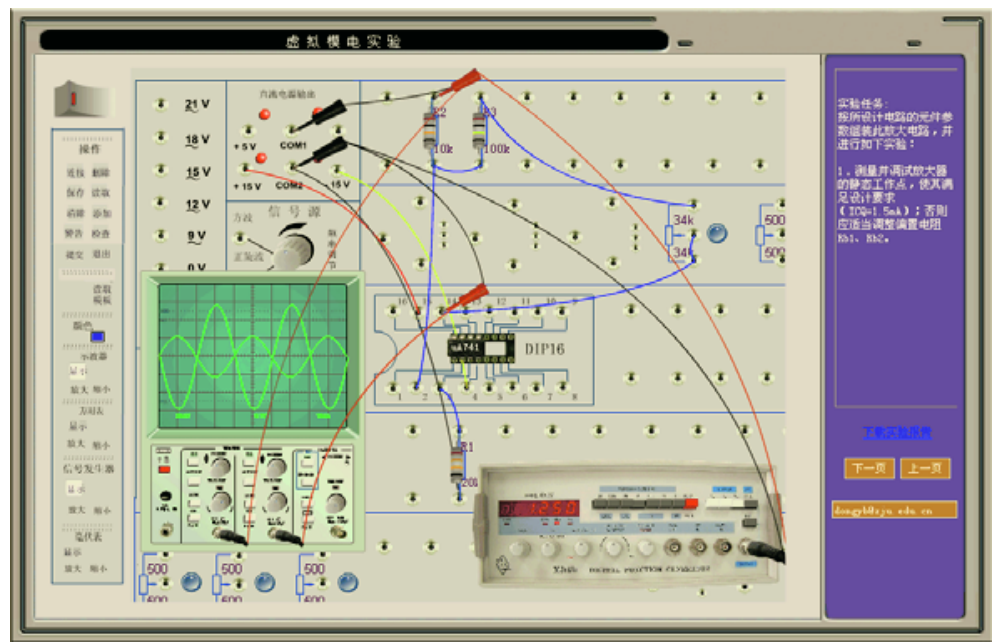

Fig. 5. Interface of ECVlab

ters of the circuit. On the right hand lists the guidance about this experiment, the user can check it anytime during the process.

Once the circuit has been constructed, user can click the power button on the left corner. The ongoing status and results sent back from server will be displayed in related virtual apparatuses. As shown in figure 5, we can clearly see the wave of the input signal and the amplified output signal on the screen of oscillograph as a result of the OPAMP. If the user adjusts the parameters of circuit or apparatus, for example, changes the signal frequency of the signal generator, the displayed result will be refreshed in real time.

The ECVlab also provides the user with convenient load and save mechanism to keep the status of the circuit which facilitates the experiment process a lot. 


\section{Implementation}

Presently, the ECVlab has been implemented and successfully applied to the students as an undergraduate course in Zhejiang University. In order to evaluate the effects, we conduct a survey among the students who have used the system for a semester. According to the results, around $87 \%$ of the students think the ECVlab is helpful to their study, concerning both theory and experiments. Figure 6 demonstrates the analysis of the survey about how does the ECVlab facilitate in Electronic Circuit study.

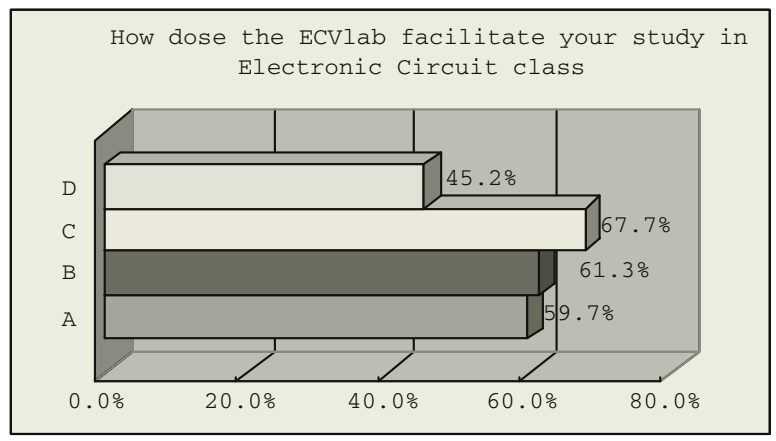

Fig. 6. Analysis of the Questionnaire

From the histogram, we can see that around $45.2 \%$ students agree that the ECVlab helps them to comprehend what they have learned from the class. Most of them $(67.7 \%)$ mention that by using the ECVlab, they can improve their capability of operation and experiment. While $61.3 \%$ students consider it as a good way to improve their interests in digital circuit. Around $59.7 \%$ think the ECVlab offers them opportunity to inspire innovation and exploration. Altogether, up to 95\% students hold positive attitude towards the ECVlab.

\section{Conclusions and Future work}

Web-based education is becoming more and more popular. In this paper, we described the design and implementation of a web-based Vlab system for Electronic Circuit simulation. This ECVlab demonstrates not only a successful integration of web servers, simulation servers, database servers, and flash-based client, but also a successful exportation to the great potential of web-based Vlab in webbased education. It can help the students to learn electronic circuit simulation by using the following features:

- Real impression and easy operation of the experiment environment.

- Immediate execution of the simulation process and direct access to the results. 
- Viewing and editing the circuit template through convenient saving and loading mechanism

- No harm to the experimental equipment compared with conducting in laboratory.

The design and implementation of the ECVlab is open to improvement. The virtual environment for digital circuit is under construction which is based on the XSPICE simulator engine. The main difference between analog circuit and digital circuit is the simulation mode. In digital circuit, the circuit state is in a incessant mode and the simulator engine needs to continuously receive circuit data and calculate the result, thus to design a arithmetic for incessant simulation is essential to our future work. In the future we will also focus on developing the performance of the system, to name a few:

- ECVlab management system including experiments management, online user management and analysis of experiment results

- New load balancing algorithms based on load average, I/O statistics.

With rapid development of Grid computing and Web services in resent years, we plan to add data grid and web services to the ECVlab in future.

Acknowledgement. The author would like to thank all the colleagues in this project. The supported of this project by a grant from the National Key Technologies R \& D Program of China is also gratefully acknowledged.

\section{References}

1. John C.Waller and Natalie Foster. Training via the web: A virtual instrument. ComputersE Education, pages 161-167, 2002.

2. Jasna Kuljis and Ray J.Paul. A review of web based simulation:whither we wander. Proceedings of the 2000 Winter Simulation Conference, pages 1872-1881, 2000.

3. Cheng K.W.E and Chan C.L. Virtual laboratory development for teaching power electronics. Power Electronics Specialists Conference ,IEEE 33rd Annual, pages 461 - 466, 2002.

4. P.Fishwick. Web based simulations: Some personal observations. Proceedings of the 1996 Winter Simulation Conference, pages 772-779, 1996.

5. X.Huang and J.Miller. Building a web based federated simulation system with jini and xml. Proceedings 34th AnnualSimulation Symposium, pages 143-150, 2001.

6. Xiaorong Xiang Yingping Huang and Gregory Madey. A self manageable infrastructure for supporting web based simulation. Proceedings of the 37th Annual Simulation Symoposium, pages 149-156, 2004. 